\title{
Virtual single-photon transition interrupted: Time-gated optical gain and loss
}

\author{
Jens Herrmann, ${ }^{1,}$ Matthias Weger, ${ }^{1}$ Reto Locher, ${ }^{1}$ Mazyar Sabbar, ${ }^{1}$ Paula Rivière, ${ }^{2,3}$ Ulf Saalmann, ${ }^{3}$ Jan-Michael Rost, ${ }^{3}$ \\ Lukas Gallmann, ${ }^{1}$ and Ursula Keller ${ }^{1}$ \\ ${ }^{1}$ Physics Department, ETH Zurich, CH-8093 Zürich, Switzerland \\ ${ }^{2}$ Departamento de Química, Universidad Autónoma de Madrid, ES-28049 Madrid, Spain \\ ${ }^{3}$ Max Planck Institute for the Physics of Complex Systems, D-01187 Dresden, Germany
}

(Received 2 July 2013; published 29 October 2013)

\begin{abstract}
The response of matter to an optical excitation consists essentially of absorption and emission. Traditional spectroscopy accesses the frequency-resolved and time-integrated response, while the temporal evolution stays concealed. However, we will demonstrate here that the temporal evolution of a virtual single-photon transition can be mapped out by a second pulsed electromagnetic field. The resulting optical signal shows optical gain and loss, which can be gated and controlled via the relative delay of the electromagnetic fields.
\end{abstract}

DOI: 10.1103/PhysRevA.88.043843 PACS number(s): 42.50.Hz, 32.80.Fb, 42.50.Nn, 78.47.jb

\section{INTRODUCTION}

The development of transmission as well as absorption spectroscopy has been crucial for understanding some of nature's secrets. In particular the laser opened the door to a variety of new spectroscopic techniques. One of these techniques, namely, femtosecond time-resolved spectroscopy, had a major impact in physics, photonics, and chemistry [1]. Most recently, time-resolved transient absorption spectroscopy was applied in the attosecond and extreme ultraviolet (XUV) regime for the first time, benefiting from the rapid progress in attosecond pulse generation [2-10]. Absorption through resonant excitation of bound-bound transitions and direct ionization plays a key role in these experiments and recent theoretical work [11-18]. On the other hand, the response of matter to off-resonant electromagnetic radiation was not considered in previous attosecond transient absorption studies, since matter is essentially transparent in this particular case. However, we will show that the presence of a second control field leads to the manifestation of gain and loss even in the time-integrated response of rather general physical systems.

We investigate the simplest model for laser-matter interaction, a two-level system (TLS). The knowledge of the temporal evolution of the TLS's dipole response is essential for the complete understanding of light-matter interaction. This evolution is nonobservable in common optical experiments, since they access only the time-integrated dipole response (TIDR). The insight into the temporal evolution with the help of time-frequency analysis allows us to develop a method to access the nonzero contributions by manipulating the evolution before it is finalized. We apply a second electromagnetic field to interrupt the evolution and unbalance positive and negative contributions, which always are present in the intrinsic temporal response (see Fig. 1). The resulting nonzero TIDR can be experimentally accessed as optical gain and loss. Furthermore, the amount of loss or gain can be controlled via the relative delay between the two electromagnetic fields. By not relying on any system-specific parameters, our formulation reveals fundamental properties of off-resonant light-matter

\footnotetext{
*jens.herrmann@phys.ethz.ch
}

interaction where the matter part is modeled with a universal TLS [19].

\section{THEORETICAL MODEL}

In our study presented here, an excitation pulse perturbatively couples the ground state to an electronic shell in an atom, which is nearly resonant. First, we show that under reasonable assumptions this situation can be described by a driven TLS with a controllable level spacing. We assume that the excited states, labeled as $|s\rangle,|p\rangle$, and $|d\rangle$, are degenerate with the same transition energy $\Delta$ relative to the ground state $|g\rangle$. For the case of helium, which we will examine experimentally further below, the corresponding states are $1 s 3 s, 1 s 3 p, 1 s 3 d$, and $1 s^{2}$, respectively. The Hamilton operator of this system reads (atomic units are used)

$$
\begin{aligned}
H= & \sum_{j=s, p, d} \Delta|j\rangle\langle j|+[\widetilde{V}(t)|g\rangle\langle p|+\bar{V}(t)| s\rangle\langle p| \\
& +\bar{V}(t)|p\rangle\langle d|+\text { H.c. }],
\end{aligned}
$$

where the ground state (with energy $E_{g}=0$ ) couples mainly via the excitation pulse (with frequency $\widetilde{\omega} \approx \Delta$ ) to the state $|p\rangle$. The excitation pulse is denoted by $\widetilde{V}(t)$, which is the product of the dipole matrix element $d_{g p}$ and the electric field of the excitation pulse, i.e., $\widetilde{V}(t)=d_{g p} \widetilde{E}_{t} \cos (\widetilde{\omega} t)$ with a Gaussian envelope $\widetilde{E}_{t}=\widetilde{E}_{0} e^{-2 \ln 2(t / \widetilde{T})^{2}}$. The second electromagnetic field, denoted by $\bar{V}(t)$, can be neglected here but is the dominant term for the coupling elements between $|p\rangle$ and the other excited states $|s\rangle$ and $|d\rangle$, respectively. Under these assumptions the part of $H$ containing the manifold of excited states can be diagonalized through a unitary transformation, which does not depend on $\bar{V}(t)$. The transformed Hamilton operator reads

$$
\begin{aligned}
H= & \sum_{j=-1,0,+1}[\Delta+j \sqrt{2} \bar{V}(t)]|j\rangle\langle j| \\
& +\sum_{j=-1,+1}([j \tilde{V}(t) / \sqrt{2}]|g\rangle\langle j|+\text { H.c. })
\end{aligned}
$$

Apart from the "dark state" $|0\rangle$, which does not couple to the ground state, one gets the two states $| \pm 1\rangle$, for which the transition energy is modulated with the second electromagnetic 
field, the so-called control pulse. Since the interaction for the two states differs only in the sign of the control pulse and the states do not couple to each other, it is sufficient to consider only one of them. Hence, our system reduces to an effective TLS described by the time-dependent state $|\psi(t)\rangle=|g\rangle a_{g}(t)+|e\rangle a_{e}(t)$, where $|e\rangle \equiv|+1\rangle$ without loss of generality is the excited state and $|g\rangle$ the ground state. $a_{g}(t)$ and $a_{e}(t)$ correspond to the amplitudes of the ground and excited states, respectively. Since the driving by the excitation pulse is perturbative we may assume $a_{g}(t) \equiv 1$. Thus we arrive at the Schrödinger equation for the amplitude $a_{e}$ :

$$
i \frac{\partial}{\partial t} a_{e}(t)=[\Delta+\bar{V}(t)] a_{e}(t)+\widetilde{V}(t) .
$$

To simplify Eq. (3) we redefined the laser pulse to get rid of the factor of $\sqrt{2}$. Note that $\Delta+\bar{V}(t)$ may cross the energy $\widetilde{\omega}$. This, however, occurs only at certain instances in time. In a dressed-state picture [cf. the discussion around Eqs. (7) and (8)], the (dressed) excited states are off-resonant.

For the optical response we need the dipole $\boldsymbol{d}(t)=$ $a_{g}(t)\langle g|\boldsymbol{r}| e\rangle a_{e}(t)+$ c.c., which has under the aforementioned assumptions a particularly simple form, $d(t) \propto a_{e}(t)+a_{e}^{*}(t)$. Considering an ensemble of atoms (each represented by the TLS) the electric field generated by the dipole oscillation reads

$$
\boldsymbol{E}_{\mathrm{dip}}(t)=\frac{2 \pi}{c} \frac{\partial}{\partial t} \boldsymbol{P}(t)=\frac{2 \pi}{c} n l \frac{\partial}{\partial t} \boldsymbol{d}(t),
$$

with the polarization $P$ due to a collection of dipoles with density $n$ in a volume of length $l$ [20]. This equation shows that the (time derivative of the) dipole $\boldsymbol{d}$ determines the measured intensity of the electric field

$$
\begin{aligned}
S(\omega) & =\left|\int d t e^{i \omega t}\left[\boldsymbol{E}_{\text {pulse }}(t)+\boldsymbol{E}_{\text {dip }}(t)\right]\right|^{2} \\
& =\left|\boldsymbol{E}_{\text {pulse }}(\omega)\right|^{2}+2 \operatorname{Re}\left[\boldsymbol{E}_{\text {pulse }}(\omega) \cdot \boldsymbol{E}_{\text {dip }}^{*}(\omega)\right]+\left|\boldsymbol{E}_{\text {dip }}(\omega)\right|^{2} .
\end{aligned}
$$

The expression applies under the assumption that the pulses can be considered as plane waves and that propagation effects can be neglected [20], which is the case for dilute gases. Obviously, in order to understand the measured optical response for atoms it is sufficient to consider the time-dependent dipole response $d(t)$ along the laser-polarization axis.

With the formal solution of the Schrödinger equation in the absence of a control field and the slowly varying envelope approximation for the excitation pulse, the dipole of the TLS in the rotating-wave approximation is given by

$$
d(t)=\frac{1}{\widetilde{\omega}-\Delta} \widetilde{V}(t)
$$

for off-resonant excitation, i.e., $|\tilde{\omega}-\Delta| \tilde{T} \gg 1$. Recall that $\tilde{T}$ represents the pulse duration of the exciting pulse. The dipole response follows the electric field $\widetilde{V}(t)$ in this case and is shown in Fig. 1(a). The response intrinsically comprises positive and negative contributions of comparable magnitude. Nevertheless, it vanishes upon temporal integration [Fig. 1(b), solid blue line] and is nonaccessible in traditional spectroscopy. Additionally, we plot the TIDR when integrated only over
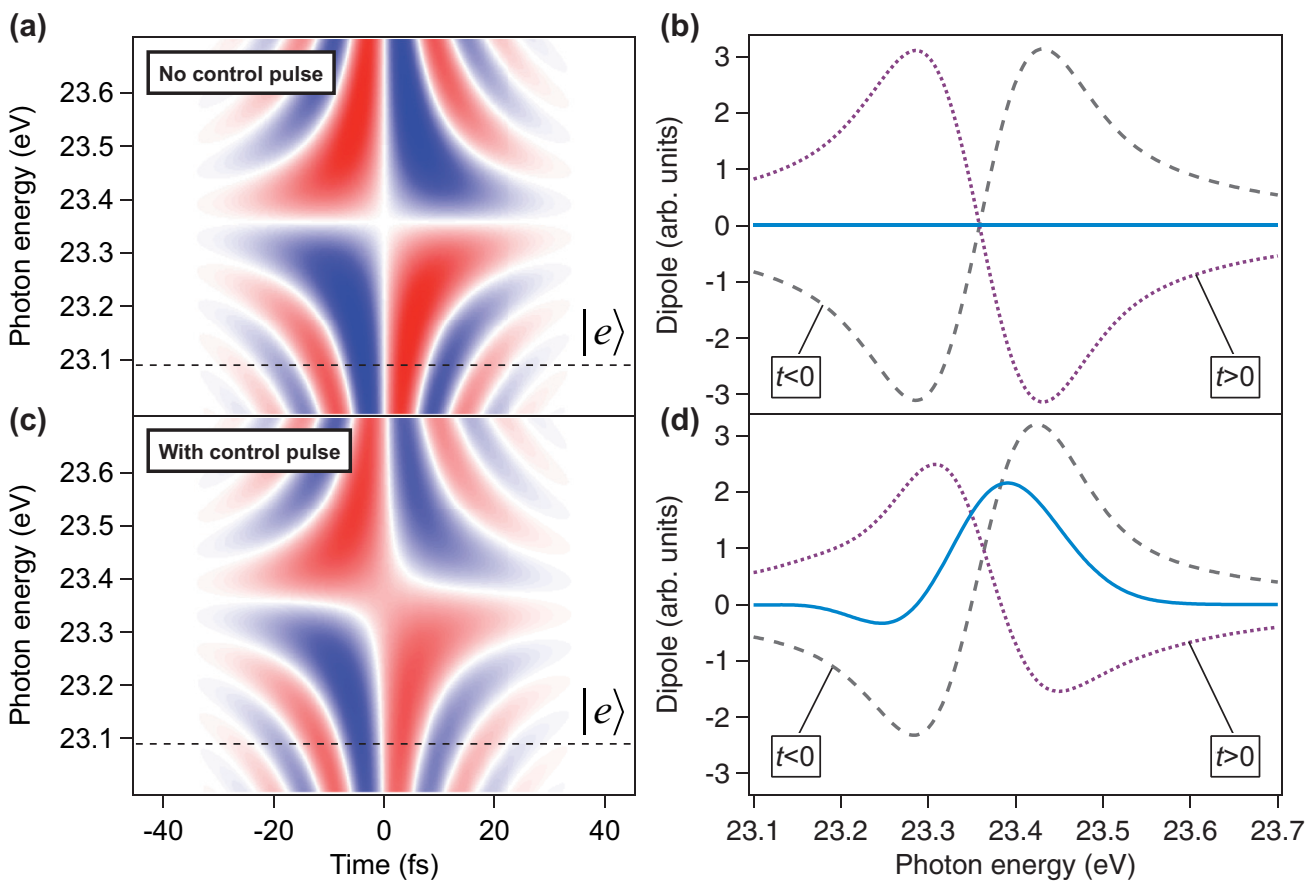

FIG. 1. (Color online) Time-frequency analysis of the dipole response of the two-level system for off-resonant excitation. (a) depicts the time-frequency representation of the dipole response. Red represents a positive and blue a negative response. In (a) and (c) the dashed line shows the energetic position of the excited state $|e\rangle$. (b) shows the time-integrated dipole response for negative times (dashed gray) and positive times (dotted purple). They differ only in the sign, which results in a zero TIDR when integrating over positive and negative times (solid light blue). (c) presents the dipole response with a loss term added to the model and a control pulse arriving 15 fs after the exciting pulse. The dipole response for positive times (dotted purple) in (d) is significantly reduced. Therefore, the temporal integration of the dipole response (solid light blue) is nonzero with positive and negative contributions. 
negative or positive times in Fig. 1(b). This illustrates how the positive- and negative-time contributions cancel each other out.

Now we show how to resolve and control this fundamental process in time by adding a control pulse to modulate the system. This results in real emission and absorption.

For an oscillatory control field $\bar{V}(t)=\bar{V}_{0} \cos (\bar{\omega} t)$ the dipole is given as an infinite sum:

$$
d(t)=\left(\sum_{k=-\infty}^{+\infty} \frac{\left[J_{k}\left(\bar{V}_{0} / \bar{\omega}\right)\right]^{2}}{\widetilde{\omega}-(\Delta+k \bar{\omega})}\right) \widetilde{V}(t)
$$

with the $J_{k}$ denoting Bessel functions of the first kind. Clearly, the response is given as a sum over weighted contributions of dressed states that are separated from the original state by multiples of the infrared (IR) frequency $\bar{\omega}$. If the instantaneous amplitude of the infrared pulse changes adiabatically in time, one can replace $\bar{V}_{0}$ in the argument of the Bessel functions by the infrared envelope $\bar{V}_{t}$ to get the time-dependent dipole for a finite control pulse.

The simplest way to account qualitatively for higher excited or continuum states within the TLS is to introduce a loss term into the Schrödinger equation (3). This term is chosen to be proportional to the instantaneous infrared field, since this field dominates the coupling. The parameter $\gamma$ defines the overall coupling strength. The consequence is a broadening of the transition, which changes the expression for the dipole response [see Fig. 1(c)] to

$d(t) \approx\left(\sum_{k=-\infty}^{+\infty} \frac{\tilde{\omega}-(\Delta+k \bar{\omega})}{[\widetilde{\omega}-(\Delta+k \bar{\omega})]^{2}+\left[\gamma \bar{V}_{t}\right]^{2}}\left[J_{k}\left(\bar{V}_{t} / \bar{\omega}\right)\right]^{2}\right) \widetilde{V}(t)$.

The actual pulse parameters of $23.37 \mathrm{eV}$ center energy and 20 fs duration for the exciting pulse and $1.57 \mathrm{eV}$ and 30 fs duration for the control pulse are in principle arbitrary, but chosen here to match our experiment as further discussed below. The intensity of the moderately strong control pulse was chosen such that it was strong enough to periodically modulate the resonance energy (which elsewhere has been referred to as Stark modulation [21]) of the TLS but sufficiently weak to avoid multiphoton excitation.

Figure 1(c) displays the dipole response of the TLS for the off-resonant excitation pulse overlapping the control pulse with a lead of $15 \mathrm{fs}$. For times $t>0$ the dipole response is significantly suppressed, resulting in a nonzero TIDR. A positive response represents absorption, while a negative response corresponds to a net emission of photons (red and blue in the color scale of Fig. 1, respectively). We interpret the net emission as optical gain since the photon count in the affected spectral regions is increased with respect to the spectrum of the incident pulse. Integration over the full spectrum yields no net emission of photons, due to energy conservation. We expect a similar response for quite diverse systems with appropriate intensity and photon energy scaling since our model does not rely on any system specific property. (a)

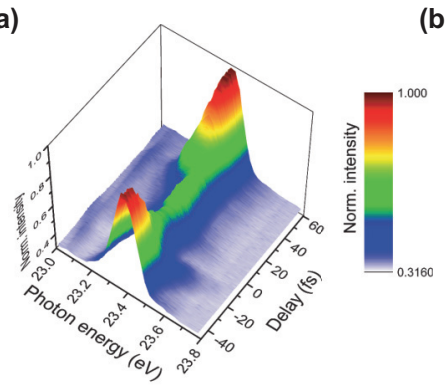

(b)

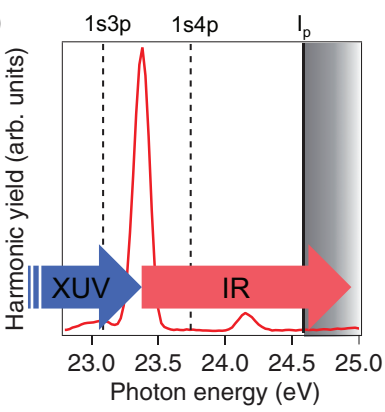

FIG. 2. (Color online) Investigated spectral window and transmitted XUV signal for different delays between the XUV and IR pulse. (a) The transmitted XUV signal shows strong absorption (about $50 \%$ ) when both pulses overlap ( $\sim 0$ fs delay) due to a two-photon absorption [also shown in (b)]. (b) The 15th harmonic is energetically located between the excited $1 s 3 p$ and $1 s 4 p$ states of He. Additionally the energy is below the ionization potential of $\mathrm{He}(24.59 \mathrm{eV})$. The small peak at $24.2 \mathrm{eV}$ is an effect due to higher-order diffraction of the grating's spectrometer.

\section{EXPERIMENTAL SETUP}

We tested our model by comparing it with a pump-probe experiment. For the TLS we chose a quantum-mechanical prototype system: helium $(\mathrm{He})$ in its $1 s^{2}$ ground state. The $1 s 3 p$ state represents the excited state $|e\rangle$. We used an attosecond pulse train (APT) for the off-resonant excitation. The APT was created by focusing the output of a Ti:sapphire amplifier system (1 kHz, $1.2 \mathrm{~mJ}, 796 \mathrm{~nm}, 30 \mathrm{fs})$ into an Ar-filled gas cell. The spectrum of the APT is composed of discrete peaks at odd multiples of the generating laser pulse carrier frequency [6]. The 15th harmonic has a central energy of $23.37 \mathrm{eV}$ (FWHM $\sim 130 \mathrm{meV}$ ), well below the ionization potential of $\mathrm{He}(24.59 \mathrm{eV})$ [22]. The 15th harmonic was off-resonant with respect to the $1 s 3 p$ and $1 s 4 p$ states. The use of a single attosecond pulse is not possible in our case. The broad continuous spectrum would simultaneously populate several excited states and prevent an off-resonant excitation. An aluminum filter with a thickness of $150 \mathrm{~nm}$ removes the residual IR radiation after the generation of the APT. Additionally, the filter compresses the APT in time due to its negative dispersion in this spectral region [23]. A temporal characterization of the APT with the technique of reconstruction of attosecond beating by interference of two-photon transitions [6,24] gives us an average duration of $\sim 350$ as for a pulse in the APT. The duration of the isolated harmonic 15 can be estimated to about $12 \mathrm{fs}$, which was derived from a previous experiment [4]. Figure 2(b) depicts the investigated spectral window, with the relevant states and the ionization threshold. We neglected the $1 s 4 p$ state, since the dipole matrix element for the transition from the ground state is by a factor of 3 smaller compared to the $1 s 3 p$ state [25].

\section{EXPERIMENTAL RESULTS AND DISCUSSION}

For the control pulse we used a moderately strong IR pulse with an intensity of $4.2 \times 10^{12} \mathrm{~W} / \mathrm{cm}^{2}$, which was separated from the driving IR pulse before the generation of the APT (from now on referred to as the XUV pulse) and sent on an independent beam path. This path length can be controlled in 
order to set the timing between the XUV and the IR pulses. This enables us to vary the relative delay between the two pulses, which allows one to control absorption and emission in the time domain in a fundamentally different way compared to former investigations [26-28]. A mirror with a center hole collinearly recombines the XUV and IR pulses. A toroidal mirror focuses both beams, IR and XUV, into the pulsed interaction target. The target works with the same repetition rate as the laser and is synchronized to the arriving pulses. The interaction length in the target is $1 \mathrm{~mm}$. This leads to an absorption of $\sim 50 \%$ (corresponding to a particle density of $\sim 5 \times 10^{17}$ particles $/ \mathrm{cm}^{3}$ ) [4] of those harmonics that are located energetically above the ionization threshold of helium $(24.59 \mathrm{eV})$. A spherical mirror that creates an astigmatic focus collects the transmitted XUV radiation. The resulting vertical line focus is well matched to the entrance slit of our grating spectrometer ( $\sim 30 \mathrm{meV}$ resolution), where we detect the XUV radiation with a CCD camera. We filter out the IR control pulse with an additional aluminum filter between the interaction target and the spectrometer.

Figure 2(a) displays the transmitted XUV yield for different delays between the two pulses. The transmitted signal exhibits a strong reduction when the peak of the XUV and IR pulses coincide in the target around $\sim 0$ fs delay. Since the 15th harmonic is off resonant and energetically below the ionization potential of $\mathrm{He}$, absorption occurred only through two-photon absorption with an XUV photon and an assisting IR photon by exciting an electron into the continuum via a virtual dipole transition [29]. To analyze the pump-probe data we plotted the change in absorbance $\Delta \alpha=\ln (\widetilde{I} / I)$ as induced by the IR gating pulse in Fig. 3(a). Here, $I$ and $I$ represent the harmonic signal without and with the IR pulse, respectively. Positive $\Delta \alpha$ indicates absorption and is displayed in red. The strong decrease of the transmitted signal related to the two-photon absorption via the virtual state is still evident. It is energetically located around the center energy of the 15th harmonic. As predicted in the model, we also observed a net emission of XUV photons, which corresponds to a negative $\Delta \alpha$ (blue). The strength of the optical gain is of the same order of magnitude as the absorption and can be controlled over the relative delay between the two electromagnetic fields. We detected optical gain below $23.2 \mathrm{eV}$ and above $23.5 \mathrm{eV}$. In these spectral regions the original pulse had no significant contributions. Additionally, since we off-resonantly excite our system and do not create a coherence we exclude perturbed free-induction decay as the origin of the effect we observe [30].

Figure 3(b) shows the theoretically predicted behavior for different time delays between the two electromagnetic fields. A negative delay corresponds to the control pulse arriving before the excitation pulse. This delay-frequency representation of the TIDR is a fundamentally different quantity compared to the time-frequency analysis of the dipole response in Fig. 1. It is constructed by time integration of the dipole response for varying delay between the two interacting pulses. The simulated pump-probe data show that for certain pulse delays a strong positive or negative dipole response, i.e., optical loss or gain, remains after the temporal integration. The TIDR is symmetric around 0 fs delay and point symmetric about the center frequency of the excitation

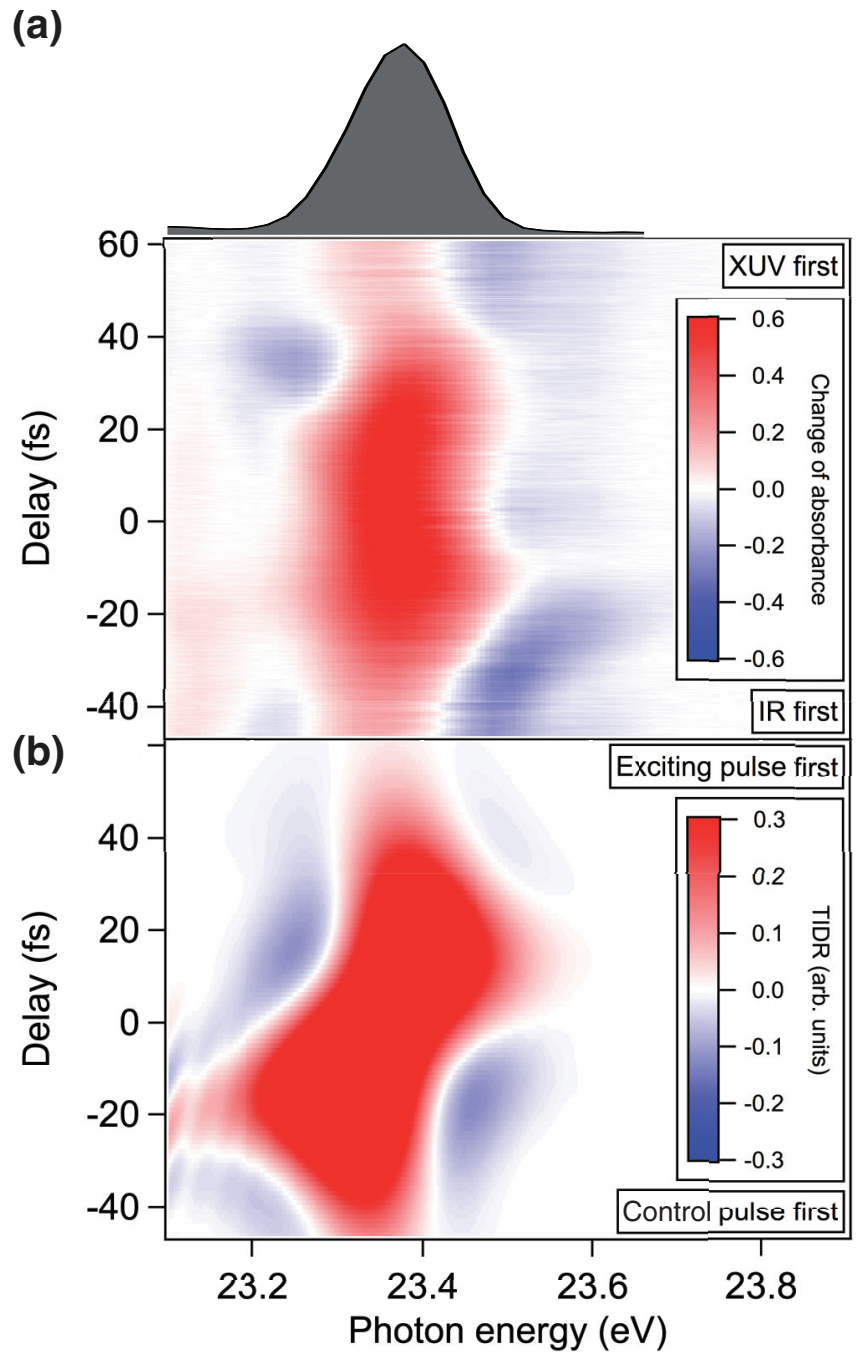

FIG. 3. (Color online) Change of absorption (a) and calculated time-integrated dipole response (b) for different delays between the pulses. The change in optical density at XUV frequencies is plotted for different delays between the two pulses. For positive delays the XUV pulse is preceding the IR pulse. Red corresponds to a positive dipole response and is related to absorption, whereas blue stands for a negative dipole response (net emission, optical gain). On top of (a) the spectrum of the XUV pulse is shown. We also detect significant optical gain in spectral regions where the intensity of the original XUV pulse signal has already decreased to the noise level.

pulse. The relative delay between the two fields determines the magnitude and sign of the response and we can switch between absorption and gain in a given spectral window. In perfect agreement with our experimental results, we observe a negative TIDR, i.e., optical gain, for negative and positive delays below and above the center energy of the excitation XUV pulse.

The behavior we observed is generic for any TLS and the underlying time-domain control mechanism offers an intuitive way for the creation and control of optical gain in this spectral region. We expect our universal model to be applicable in entirely different classes of systems ranging from molecules to low-dimensional solid-state systems in case of proper scaling of photon energies and intensities. 


\section{ACKNOWLEDGMENTS}

This work was supported by the National Center of Competence in Research Molecular Ultrafast Science and Technology (NCCR MUST), research instrument of the Swiss
National Science Foundation. P.R. acknowledges a Juan de la Cierva Contract Grant from MICINN, and the COST Action CM0702. We thank H. R. Reiss and M. Lucchini for fruitful discussions.
[1] A. H. Zewail, J. Phys. Chem. A 104, 5660 (2000).

[2] E. Goulielmakis et al., Nature (London) 466, 739 (2010).

[3] H. Wang, M. Chini, S. Chen, C.-H. Zhang, F. He, Y. Cheng, Y. Wu, U. Thumm, and Z. Chang, Phys. Rev. Lett. 105, 143002 (2010).

[4] M. Holler, F. Schapper, L. Gallmann, and U. Keller, Phys. Rev. Lett. 106, 123601 (2011).

[5] M. Hentschel et al., Nature (London) 414, 509 (2001).

[6] P. M. Paul, E. S. Toma, P. Breger, G. Mullot, F. Augé, P. Balcou, H. G. Muller, and P. Agostini, Science 292, 1689 (2001).

[7] G. Sansone et al., Science 314, 443 (2006).

[8] R. Kienberger, E. Goulielmakis, M. Uiberacker, A. Baltuska, V. Yakovlev, U. Heinzmann, M. Drescher, and F. Krausz, Nature (London) 427, 817 (2004).

[9] L. Gallmann, C. Cirelli, and U. Keller, Annu. Rev. Phys. Chem. 63, 447 (2012).

[10] F. Krausz and M. Ivanov, Rev. Mod. Phys. 81, 163 (2009).

[11] Z.-H. Loh, C. H. Greene, and S. R. Leone, Chem. Phys. 350, 7 (2008).

[12] M. B. Gaarde, C. Buth, J. L. Tate, and K. J. Schafer, Phys. Rev. A 83, 013419 (2011).

[13] S. Chen, K. J. Schafer, and M. B. Gaarde, Opt. Lett. 37, 2211 (2012).

[14] R. Santra, V. S. Yakovlev, T. Pfeifer, and Z.-H. Loh, Phys. Rev. A 83, 033405 (2011).

[15] P. Rivière, O. Uhden, U. Saalmann, and J. M. Rost, New J. Phys. 11, 053011 (2009).
[16] S. Chen, M. Wu, M. B. Gaarde, and K. J. Schafer, Phys. Rev. A 87, 033408 (2013).

[17] M. Chini, X. Wang, Y. Cheng, Y. Wu, D. Zhao, D. A. Telnov, S.-I. Chu, and Z. Chang, Scientific Report 3, 1105 (2013).

[18] S. Chen, M. J. Bell, A. R. Beck, H. Mashiko, M. Wu, A. N. Pfeiffer, M. B. Gaarde, D. M. Neumark, S. R. Leone, and K. J. Schafer, Phys. Rev. A 86, 063408 (2012).

[19] The interaction of two laser fields with an atomic two-level system has already been studied in previous investigations; see, e.g., [26,28]. However, these studies were performed in the monochromatic limit, i.e., using continuous-wave lasers or with a fixed delay between the two laser pulses.

[20] J. C. Baggesen, E. Lindroth, and L. B. Madsen, Phys. Rev. A 85, 013415 (2012).

[21] Y. V. Radeonychev, V. A. Polovinkin, and O. Kocharovskaya, Phys. Rev. Lett. 105, 183902 (2010).

[22] G. W. F. Drake and W. C. Martin, Can. J. Phys. 76, 679 (1998).

[23] R. López-Martens et al., Phys. Rev. Lett. 94, 033001 (2005).

[24] H. G. Muller, Appl. Phys. B 74, 17 (2002).

[25] Edited by G. W. Drake, Springer Handbook of Atomic, Molecular, and Optical Physics (Springer, Berlin, 2006).

[26] B. R. Mollow, Phys. Rev. A 5, 2217 (1972).

[27] F. Y. Wu, S. Ezekiel, M. Ducloy, and B. R. Mollow, Phys. Rev. Lett. 38, 1077 (1977).

[28] J. C. Delagnes and M. A. Bouchene, Phys. Rev. A 76, 023422 (2007).

[29] M. Göppert-Mayer, Ann. Phys. (Leipzig) 401, 273 (1931).

[30] P. Hamm, Chem. Phys. 200, 415 (1995). 